\title{
Standardless Quantification Of Actinides By EPMA
}

A. Moy ${ }^{1,2}$, C. Merlet ${ }^{1}$, O. Dugne ${ }^{3}$.

${ }^{1 .}$ GM, CNRS, Université de Montpellier II, Place E. Bataillon, 34095 Montpellier, France.

2. CEA, DEN, DTEC, SGCS, LMAC, 30207 Bagnols-sur-Cèze, France.

3. CEA, DEN, DEC, SA3C, 13108 St Paul lez Durance, France.

Electron probe microanalysis (EPMA) is an analysis technique used to quantify with a high accuracy the amount of elements present on a sample of unknown composition. However, for some elements, such as the actinides, quantitative EPMA is not possible to achieve due to the lack of suitable reference standard samples. To overcome this difficulty, standardless methods can be employed with the use of virtual standards. Standardless method requires the accurate knowledge of absolute characteristic x-ray intensities. These x-ray intensities can be obtained by analytical calculations or by Monte Carlo (MC) simulations with mean of physical parameters. However, these parameters are not always well known and can lead to inaccurate results when used in standardless quantification methods. Validation by experimental measurements of the calculated x-ray intensities is needed.

Absolute $\mathrm{M} \alpha$ and $\mathrm{M} \beta \mathrm{x}$-ray intensities were measured by electron impact on thick samples of $\mathrm{Pt}, \mathrm{Au}, \mathrm{Pb}$, $\mathrm{U}$ and $\mathrm{Th}$ for energies ranging from the ionization threshold up to $38 \mathrm{keV}$. X-ray intensities were recorded with an electron microprobe using several high resolution wavelength-dispersive spectrometers and converted into absolute $\mathrm{x}$-ray yields by evaluation of the detector efficiency.

The experimental absolute $\mathrm{M} \alpha$ and $\mathrm{M} \beta \mathrm{x}$-ray yields were compared to the absolute $\mathrm{x}$-ray intensities simulated by the multi-purpose Monte Carlo code PENELOPE [1]. For the studied elements, good agreement was obtained between the experimental and the simulated data, allowing future use of the simulated $\mathrm{x}$-ray intensities in standardless quantification methods (Fig. 1).

For practical applications, the x-ray intensities of the most intense L and M x-ray lines were calculated for elements with atomic number $89 \leq \mathrm{Z} \leq 99$ and for accelerating voltage ranging from the ionization threshold up to $40 \mathrm{kV}$. For a convenient use, the data were stored in a database in function of the accelerating voltage.

To convert the virtual intensities into intensities which can be used in practical applications, the characteristics of the spectrometer used must be determined experimentally. Indeed, the simulated x-ray intensity is related to the recorded signal by the detection efficiency of the spectrometer and by the spectral broadening of the studied x-ray line.

To determine the spectrometer characteristics, an accurate method was employed [2-4]:

- The spectral broadening induced by the spectrometer and by the natural line-width is determined by non-linear fitting of the $\mathrm{x}$-ray line of interest by a set of Pseudo-Voigt functions.

- The detection efficiency of the spectrometer, which depends of the measured photon energy, is obtained by comparison between the bremsstrahlung intensity emitted by a bulk standard of carbon or nickel and the bremsstrahlung calculated by the MC code PENELOPE.

The virtual standard intensity of the studied $x$-ray line is finally given by the ratio of the calculated x-ray intensity and the normalized area of the x-ray line, timed by the spectrometer efficiency.

To facilitate the implementation of the method, a software program was developed. The interface allows the user to manage a database of pre-calculated x-ray intensities, assists during the determination of the spectrometer characteristics and calculate virtual standard x-ray intensities for the studied element. 
The virtual value can then be used as a standard value in the microprobe software to perform classical quantitative analysis.

Standardless quantifications of heavy elements, such as lead, thorium, uranium and plutonium, were tested. The tests were firstly performed on standard samples of $\mathrm{PbS}, \mathrm{PbTe}, \mathrm{PbCl}_{2}$, vanadinite, and $\mathrm{UO}_{2}$ (Fig. 2) to confirm the method. Standardless quantification results were shown in agreement with classical quantification results.

The measurements were secondly performed on actinide samples of $\mathrm{U}, \mathrm{ThO}_{2}, \mathrm{ThF}_{4}$ and $(\mathrm{U}-\mathrm{Pu}-\mathrm{Am}) \mathrm{O}_{2}$, and with results obtained during previous measurement projects [5]. Actinide quantifications obtained with the virtual standards were found in good agreements with the expected results and show deviation from the expected value below $15 \%$. This confirms the reliability of the $\mathrm{x}$-ray intensities simulated by the MC code PENELOPE and the reliability of the developed method used to determine the spectrometer characteristics.

Using this method, standardless quantification of actinide by EPMA can be obtained with a good level of accuracy without actinide material standards.

\section{References:}

[1] F Salvat et al, "PENELOPE-2012: A Code System for Monte Carlo Simulation of Electron and Photon Transport", (OECD/NEA Data Bank, Issy-les-Moulineaux, France).

[2] C Merlet and X Llovet, Microchim. Acta 155 (2006) 199.

[3] C Merlet et al, Phys. Rev. A 73 (2006) 062719.

[4] A Moy et al, J. Phys. B: At. Mol. Opt. Phys. 47 (2014) 055202.

[5] C Merlet et al, Microsc. Microanal. 14 (Suppl 2) (2008) p.1094.



Figure 1. Experimental measurements and theoretical predictions of the absolute $\mathrm{M} \alpha$ and $\mathrm{M} \beta \mathrm{x}$-ray yields of U.

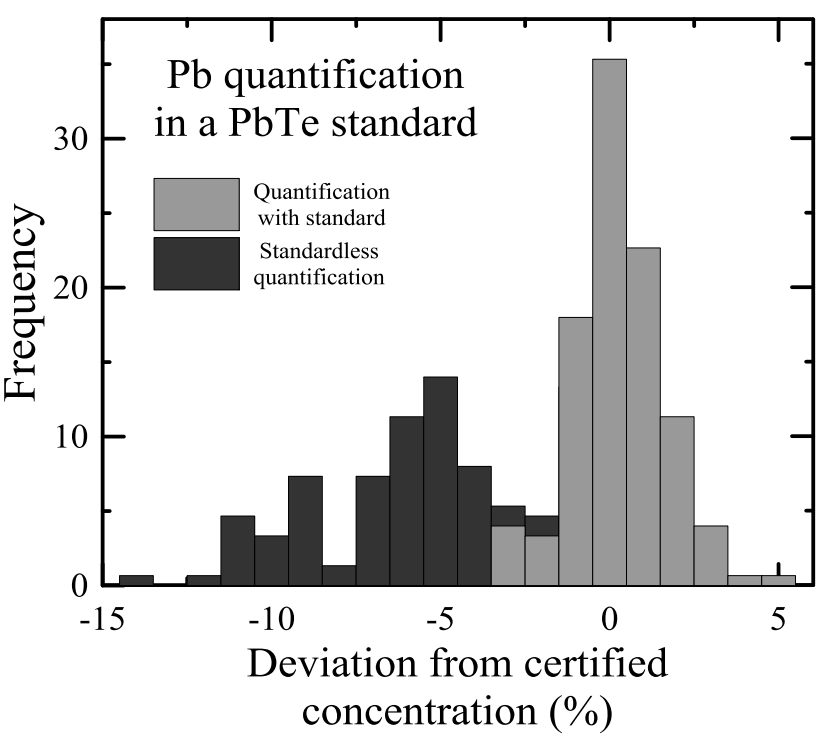

Figure 2. Comparison between standard and standardless quantification of $\mathrm{Pb}$ in a $\mathrm{PbTe}$ standard sample. 